\title{
Genetic analysis and gene cloning of a triangular hull 1 (tri1) mutant in rice (Oryza sativa $\mathbf{L}$.
}

\author{
FENG GongNeng ${ }^{1,2}$, ZHANG ChangQuan ${ }^{1}$, TANG MingYong ${ }^{1}$, ZHANG GuiYun ${ }^{1}$, \\ XU ChenWu ${ }^{1}$, GU MingHong ${ }^{1} \&$ LIU QiaoQuan ${ }^{1 *}$ \\ ${ }^{1}$ Key Laboratory of Plant Functional Genomics of Ministry of Education/Key Laboratory of Crop Genetics and Physiology of Jiangsu \\ Province, Yangzhou University, Yangzhou 225009, China; \\ ${ }^{2}$ Yancheng Institute of Technology, Yancheng 224051, China
}

Received May 16, 2012; accepted October 26, 2012; published online March 22, 2013

\begin{abstract}
Grain shape and size are two key factors that determine rice yield and quality. In the present study, a rice triangular hull mutant (tril) was obtained from the progeny of japonica rice variety Taipei 309 treated with ${ }^{60} \mathrm{Co} \gamma$-rays. Compared to the wild type, the tril mutant presents a triangular hull, and exhibits an increase in grain thickness and protein content, but with a slight decrease in plant height and grain weight. Genetic analysis indicated that the mutant phenotype was controlled by a recessive nuclear gene which is stably inherited. Using a map-based cloning strategy, we fine-mapped tril to a 47-kb region between the molecular markers CHR0122 and CHR0127 on the long arm of chromosome 1, and showed that it co-segregates with the molecular marker CHR0119. According to the rice genome sequence annotation there are six predicated genes within the mapped region. Sequencing analysis of the mutant and the wild type indicated that there was a deletion of an A nucleotide in exon 3 of the OsMADS32 gene, which could result in a downstream frameshift mutation and premature termination of the predicted polypeptide. Both semi-quantitative and real-time RT-PCR analyses showed that this gene expressed highly in young inflorescences, while expressed at very low levels in other tissues. These results implied that the OSMADS32 gene could be a candidate of TRI1. Taken together, the results of this study lay the foundation for further investigation into the molecular mechanisms regulating rice caryopsis development.
\end{abstract}

Oryza sativa L., triangular hull 1 mutant (tri1), genetic analysis, gene cloning, OsMADS32

Citation: Feng G N, Zhang C Q, Tang M Y, et al. Genetic analysis and gene cloning of a triangular hull 1 (tril) mutant in rice (Oryza sativa L.). Chin Sci Bull, 2013, 58: 2984-2991, doi: 10.1007/s11434-012-5642-9

Grain size and shape are two key determinants of cereal grain yield and quality, and are also important target traits for either domestication or genetic improvement. Rice is one of the most important foods for human beings, and also an important model for genetic studies of monocotyledonous plants. Therefore, it is quite important for both theoretical and practical reasons to elucidate the molecular mechanism of grain development. In recent years, many genes controlling rice grain size and shape have been cloned. For example, GS3 was identified as a major QTL (quantitative trait locus) controlling rice grain weight and length, and

*Corresponding author (email: qqliu@yzu.edu.cn) also as a minor QTL for grain width and thickness. GS3 might also function as a negative regulator for controlling both grain and organ size [1,2]. GS5, encoding a putative serine carboxypeptidase, plays an important role in controlling grain width, filling and grain weight. Highlevel expression of GS5 is correlated with promotion of cell division, causing an increased cell number and finally resulting in a larger grain size $[3,4]$.

The rice spikelet consists of rudimentary glumes, the lemma, palea, lodicules, stamens and pistil. Among these floral organs, development of the lemma and palea is one of the key factors controlling grain shape and size, and ultimately effect grain yield and quality. Fon (floral organ 
number) is a gene controlling rice floral organ number, which plays an important role during the development of shoot apical meristems, the inflorescence and floral meristems [5-8]. The fon mutant presents an enlargement of the floral meristem, and parts of the flowers have extra lodicules or plaea/lemma-like organs [5-8]. In 1943, Morinaga et al. [9] were the first to describe the triangular hull (TH) phenotype, subsequently, several other reports followed $[10,11]$. The triangular hull usually exhibits curved lemma, which results in the triangular phenotype of both spikelet and grain. Li et al. [11] identified a thl (triangular hull 1) mutant from a population of EMS (ethyl methane sulphonate)-induced mutants, and the $T H 1$ gene was then mapped to a $60-\mathrm{kb}$ region on chromosome 2 . The cloned $T H 1$ gene, belonging to the DUF640 domain-containing family, is mainly expressed in the young inflorescence, the lemma and palea, and has proven to be one of the most important genes controlling lemma and palea development in rice.

In the present study, we identified a tri mutant from the progency of the japonica rice variety Taipei 309 treated with ${ }^{60} \mathrm{Co} \gamma$-rays. The tri mutant is able to set seeds normally, and the mutation is stably inherited. Genetic analysis showed that the triangular hull phenotype is controlled by a single recessive nuclear gene. By using a map-based cloning strategy, the TRII gene was located within a $47-\mathrm{kb}$ region on the long arm of chromosome 1 . The results from sequence verification and gene expression revealed that OsMADS32, a gene encoding a transcription factor important for inflorescence development, could be the candidate gene for TRII.

\section{Materials and methods}

\subsection{Plant materials}

Two japonica rice varieties, Taipei 309 and Nipponbare, and one indica variety, Nanjing 11, were used in this study. The tril mutant was derived from the rice variety Taipei 309 treated with $\gamma$-rays from a ${ }^{60} \mathrm{Co}$ source.

\subsection{Investigation of agronomic traits and grain quality}

The tril mutant and the wild type (Taipei 309) were grown under normal cultural conditions during the regular ricegrowing season in the experimental field at Yangzhou University in 2010 and 2011. At maturity stage, the major agronomic traits, including plant height, tiller number, 1000grain weight, grain length, grain width and grain thickness, were carefully investigated and compared between the mutant and the wild type. The mature grains were milled and air-dried at room temperature for physical and chemical quality measurements. Total crude protein content was determined by the Micro-Kjeldahl method, and the amylose content was determined by an iodine colorimetric method. Starch viscosity was analyzed with a Rapid Visco Analyzer
(RVA) (Newport Scientific, Narrabeen, Australia) according to the manufacturer's instructions. ANOVA (an analysis of variance) was performed by using SPSS16.0 to investigate whether there was a significant difference between/ among the tested samples.

\subsection{Genetic analysis}

The tril mutant was crossed with the indica cultivar Nanjing 11 in August 2009, and the $F_{1}$ hybrid was planted in Lingshui County at Hainan Province in November of the same year. The $F_{2}$ populations were grown in the experimental field at Yangzhou University in the summers of 2010 and 2011. When the plants were mature, segregation of normal vs. triangular hull grains was determined, and the ratio was analyzed by the $\chi^{2}$-test.

\subsection{DNA extraction and molecular markers analysis}

A total of 1153 plants showing triangular hull grains and several indivduals with normal grains from the above $F_{2}$ population were used for gene mapping. DNA was extracted from the leaves by the method described by Dellaporta et al. [13]. Some SSR (simple sequence repeat) marker sequences were obtained from Gramene web (http://www. gramene.org/). Also, based on the published complete genome sequences of indica cultivar 93-11 and japonica cultivar Nipponbare, several molecular markers were designed using Primer Premier 5.0 software (Figure 1). All primers were synthesized by Shanghai Generay Biotech Co., Ltd. PCR products of SSR markers were electrophoresised on $3 \%$ agarose gels, and subsequently photographed by a Gel Imaging System after staining with ethidium bromide. For the CAPS (Cleaved amplified polymorphic sequence) marker CHR0135, the PCR product was digested with the restriction enzyme Dde I prior to electrophoresis.

\subsection{Gene mapping}

Using the principle of bulked segregant analysis (BSA), 10 normal plants and 10 mutant plants were randomly selected from the above $F_{2}$ population to construct the normal and mutant pools, respectively. Polymorphic markers were first selected between the parental lines, Nanjing 11 and Taipei 309 , and were then used to identify markers linked to the TRII gene. Based on the linked marker and the newly developed markers around the primary restricted region, fine mapping of the target gene was performed with all selected mutant plants.

\subsection{Identification of the candidate gene}

Based on the predicted gene sequences (http://blast.ncbi. nlm.nih.gov) in the fine-mapped region, specific primers were designed to amplify the puptative genes from both 
wild type and the tril mutant. The resulting PCR products were cloned into the pMD18-T vector for sequencing analysis.

\subsection{RNA extraction and expression analysis}

Different tissues, including the root, culm, leaf and leaf sheath at the elongation stage, young panicles before heading date, and developing seeds at the filling stage, were collected. Total RNAs were extracted using a total RNA extraction kit (Tiangen) according to the manufacturer's instructions. After digestion with DNase (RNase-free), the first strand cDNA was synthesized. The rice housekeeping gene OsActin1 (LOC_Os03g0718100) was chosen as the internal control and was amplified with the primer pair ACT-F (5'-CCAAGGCCAATCGTGAGAAGA-3') and ACT-R (5'-AATCAGTGAGATCACGCCCAG-3'). Semiquantitative RT-PCR was perfomed with 25 cycles of amplification. Real-time PCR was performed using the SYBR ${ }^{\circledR}$ Premi Ex Taq ${ }^{\mathrm{TM}}$ (Perfect Real-Time) Kit (TaKaRa) and the ABI 7500 Real-Time PCR system (Applied Biosystems). Each $20 \mu \mathrm{L}$ PCR reaction mixture contained $10 \mu \mathrm{L}$ of SYBR $^{\circledR}$ Premi Ex Taq $^{\mathrm{TM}}$ II $(2 \mathrm{X}), 8$ nmol of each specific forward and reverse primers, $10 \mathrm{ng}$ of the synthesized cDNA, and $0.4 \mu \mathrm{L}$ of ROX Reference Dye (50×).

\section{Results and analysis}

\subsection{Agronomic performance and grain quality of the tril mutant}

As shown in Figure 1(a) and Table 2, plant height of the tril mutant was somewhat reduced compared to the wild type
Taipei309. During panicle development, the top of the tril lemma curved inward toward the palea, some spikelets could not close completely, and the hulls exhibited the characteristic triangular shape (Figure 1(b)). After stripping off the lemma, it was seen that the palea of the wild type was much smaller, but the palea of the mutant was laterally elongated and triangular, and wrapped around most of the embryo tightly (Figure 1(d)). At maturity, the tril grains were irregular and triangular in shape (Figure 1(b)), and the brown rice was also triangular (Figure 1(c)).

Data for seed shape and size of the tril mutant and the wild type are given in Table 2. It is obvious that, compared with the wild type, grain thickness of the tril mutant increased significantly $(P<0.05)$, while the grain weight decreased significantly $(P<0.05)$. There was no significant difference observed in grain length between wild type and the mutant. Although the crude protein content of mutant grains $(8.5 \% \pm 0.1 \%)$ was significantly higher than in wild type $(6.9 \% \pm 0.2 \%)(P<0.05)$, there was no difference in the amylose content, gelatinization temperature and gel consistency (data not shown), or the RVA profile between the mutant and wild type (Figure 1(e)). This implies that mutation of the target gene had a large effect on grain shape and size, but little effect on rice grain qualities.

\subsection{Genetic analysis and mapping of the mutated gene}

$F_{2}$ seeds derived from selfing $F_{1}$ plants of the cross between Nanjing 11 and the mutant tril were all normal and basically identical to those of the wild type, which suggested that the triangular hull is a recessive trait. Among the 200 plants randomly selected from the $\mathrm{F}_{2}$ population, 157 and 43 plants exhibited the normal or tril mutant phenotypes, respectively.

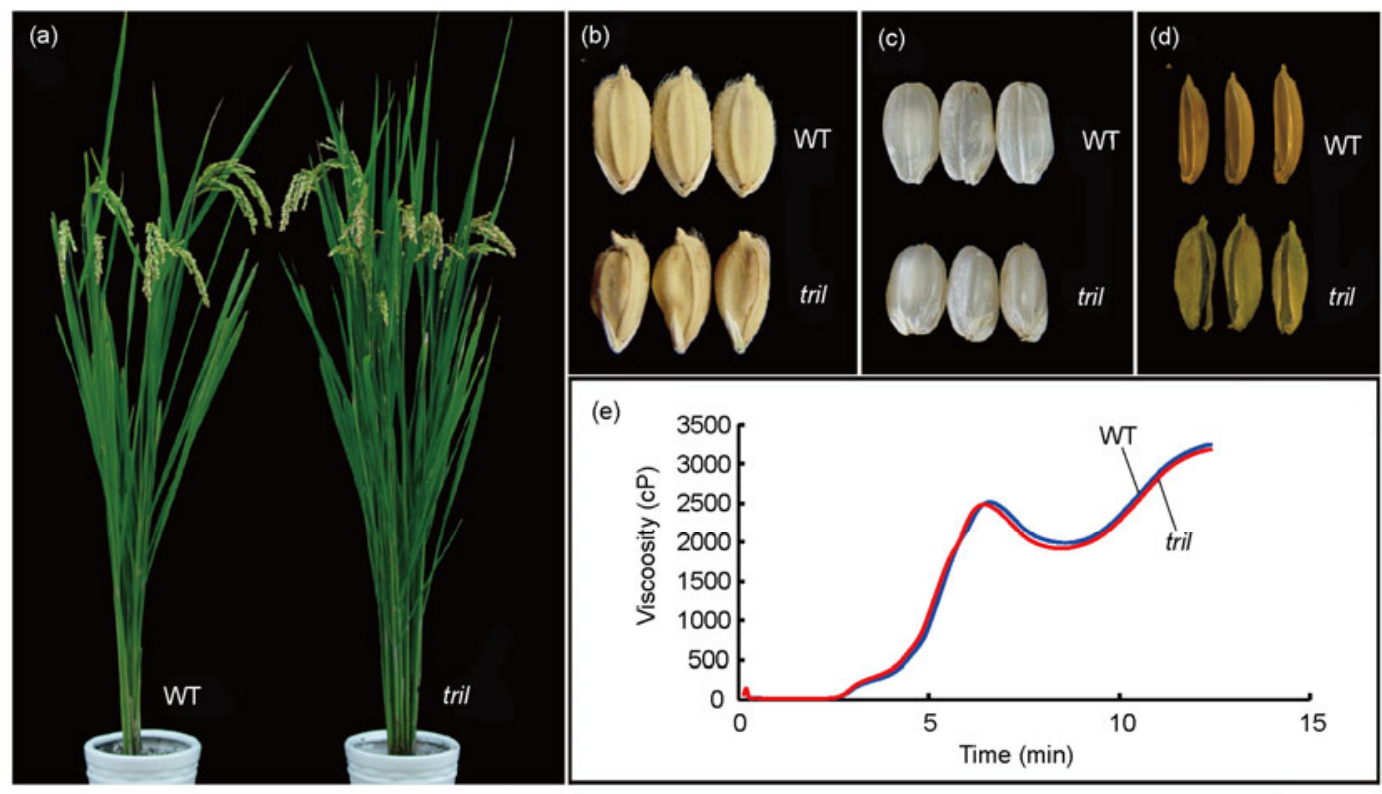

Figure 1 Phenotypic comparisons between the tril mutant and the wild type. (a) Plant phenotype; (b) mature grain; (c) brown rice; (d) palea; (e) RVA profile of milled rice. WT, wild type; tril, mutant. 
This ratio correlated well with the expected ratio of $3: 1\left(\chi^{2}=\right.$ $\left.1.31<\chi_{0.05}^{2}=3.84\right)$. Therefore, the mutant phenotype is probably caused by a recessive nuclear gene.

Among the 150 SSR markers distributed evenly on 12 chromosomes, 80 markers were identified that were polymorphic between the two parental lines, Nanjing 11 and Taipei 309. The polymorphic markers were then analyzed within both the normal and tril mutation DNA pools, and the results showed that the SSR marker CHR0101, which maps to a locus on the long arm of chromosome 1, could be linked to the target gene TRI1. Subsequently, we found that the CHR0101 marker showed significant segregation distortion among 46 mutant (tril) plants from the $\mathrm{F}_{2}$ population, but do high exchange frequency among 20 normal plants. These results implied that the CHR0101 marker is linked to the target gene.

For fine mapping, 16 new SSR or CAPS markers, from the region around the marker CHR0101, were developed by using the genome sequences of the japonica line Nipponbare and the indica line 93-11; 8 of the markers (Table 1) showed polymorphisms between Nanjing 11 and Taipei 309 . By using another 83 mutant plants from the $\mathrm{F}_{2}$ population, TRII was then mapped within a 705-kb region between the molecular markers CHR0101 and CHR0111, and it cosegregated with the markers CHR0115, CHR0116 and CHR0117 (Figure 2(a)). To further map the TRI1 gene, we generated a large $F_{2}$ population and screened 1153 mutant plants, and developed 11 new polymorphic markers (Table 1) that mapped to the interval between marker loci CHR0101 and CHR0111. The TRII gene was finally narrowed down within a 47-kb region between markers CHR0122 and CRH0127 in the BAC clone AP003343 on chromosome 1 (Figure 2(c)), and it co-segregated with the marker CHR0119 (Figure 2(b)).

\subsection{Cloning and sequence analysis of the candidate gene}

Based on the Rice Genome Annotation Project, six candidated genes identified within the mapped $47-\mathrm{kb}$ region (Figure 2(c)) are LOC_OsO1g52680, LOC_OsO1g52690, LOC_Os01g52700, LOC_OsO1g52710, LOC_OsO1g52720 and LOC_OsO1g52730. Because the partial sequences of the predicted gene LOC_OsO1g52730 were not included in the region, only the other five intact annotated genes were analyzed. The five candidate genes encode $O s M A D S 32$, a putative retrotransposon protein, a hypothetical protein, a putative glycosyl transferase 8 domain-containing protein, and a putative complex I intermediate-associated protein 30 domain-containing protein. Among these, OsMADS32 belongs to the MADS-box family and has a MIKCc type-box; this gene has been reported to be a transcription factor with a function in flower development.

Based on the gene annotation sequences of Nipponbare, specific primers were designed to amplify and clone all five genes from both the tril mutant and the wild type. The results from sequence alignment revealed that there was no difference in the sequences of four genes, $L O \mathrm{C}_{-}$ Os01g52690,LOC_Os01g52700,LOC_OsO1g52710 and LOC_OsO1g52720 between the mutant and the wild type. However, a single nucleotide A deletion was identified in the third exon of the gene LOC_OsO2g56610 (OsMADS32) in the mutant compared with the wild type (Figure 2(d)). This deletion is predicted to result in a frameshift at the 107 th codon and then premature termination at the 109th codon (Figure S1), which could cause a change or loss of

Table 1 Primer sequences for the molecular markers developed in this study

\begin{tabular}{|c|c|c|}
\hline Molecular marker & Forward primer $\left(5^{\prime} \rightarrow 3^{\prime}\right)$ & Reverse primer $\left(5^{\prime} \rightarrow 3^{\prime}\right)$ \\
\hline CHR0101 & ATAGTTCGCCATCGTCAT & ACACGCCATAGCAAGGAA \\
\hline CHR0102 & CATATCTCTACAAAАCAAACAAAAA & CATCAATGGTGGTGACCTTTT \\
\hline CHR0103 & CACATTTTCTGTCCCAAAGTTCA & ATTTAGAGCGTGTTGTGTTTTGG \\
\hline CHR0107 & ААССССТСССТТСТССТТСТТС & CACAGGACGTGAGGCTCGG \\
\hline CHR0111 & TCCTCCTCATTCGGACACTTTT & CCAAACAGTGGATGGAGCAGTAG \\
\hline CHR0114 & GCACATTTTATTTCGATTCGACCA & ATCTTGCCCCTTTCTCGCCT \\
\hline CHR0115 & AATCTGTATGGTCAACTCTTCAC & AGCAGCCTAACTTATGTTCCA \\
\hline CHR0116 & AGAGGAAATGATGGTCTGGAG & GTACATAGGCAGAAGCTCTAAAGTC \\
\hline CHR0117 & GTGTTAAGGCTTGTCCCGAGT & АATCTCCCAATCTCGTAGGCT \\
\hline CHR0119 & CCAATGGTAGGTGAAACAAAGAATG & TACAGGCTCTCGTCTTCAACACACT \\
\hline CHR0120 & AGGAGAGGAGAGACGCTGGCT & TACAGCAACCCCCCAAATCAT \\
\hline CHR0122 & CTCTTGCCGCAAACACTTCT & CAGATTTCCTTTATTGCTGCTTAG \\
\hline CHR0127 & TGTGGGTAAGTTCTGACGGGATT & GCACGCAAACGACGAAACAG \\
\hline CHR0128 & TTGATGTAGCGGATGATGGCGTTA & CAGCCAATGCCTGCCGAAAT \\
\hline CHR0135 & CGTAGTAATCAGTTGTTTGAGCGTT & CACAGGTATGAATCGTATCAGAAGT \\
\hline
\end{tabular}




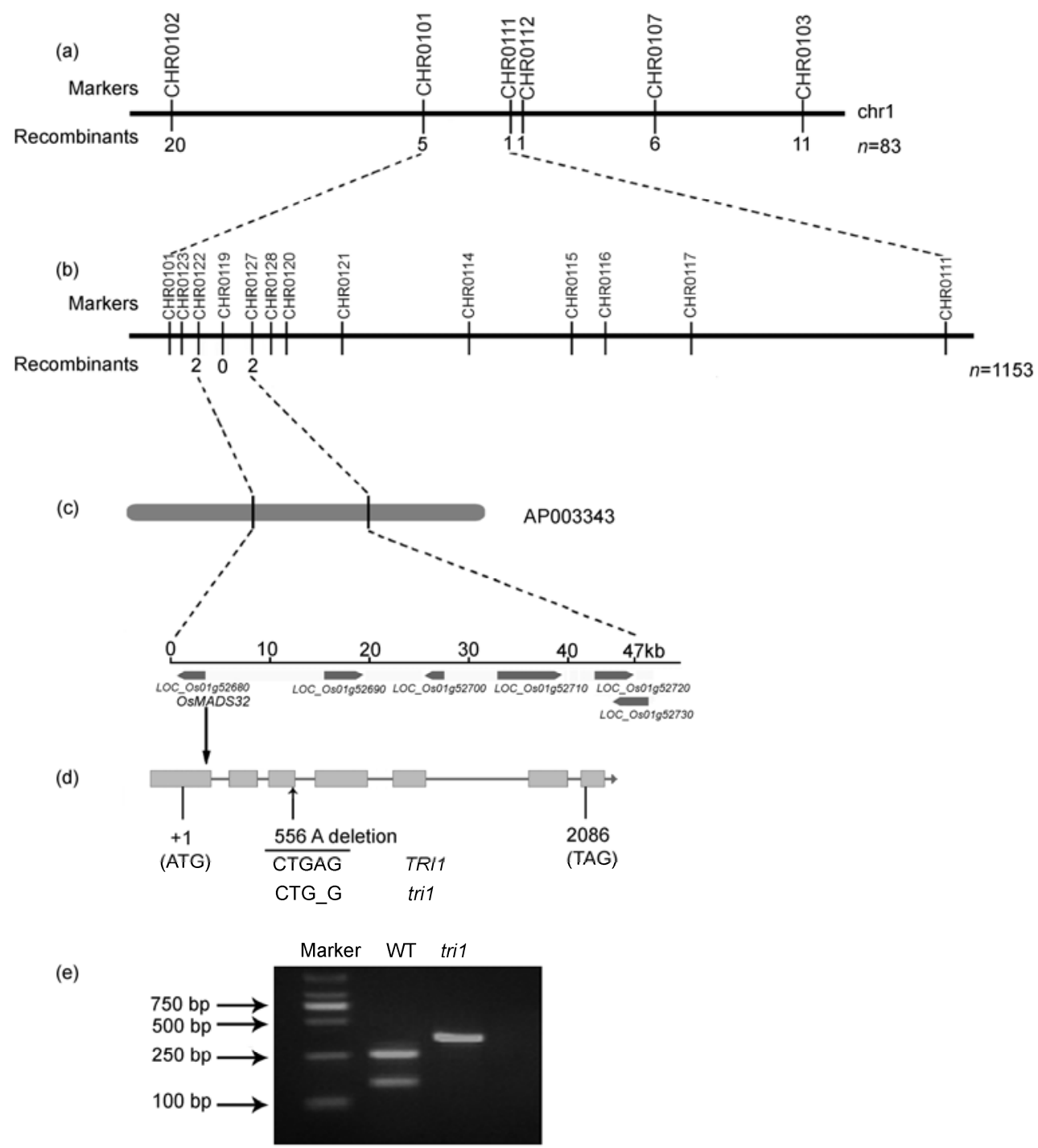

Figure 2 Gene mapping of TRII on the long arm of chromosome 1 and candidate gene prediction. (a) Primary mapping of TRII on chromosome 1 between the markers CHR0101 and CHR0111; (b) fine mapping of TRII between markers CHR0122 and CHR0127 in a 47-kb region; (c) the mapped region is located in the BAC clone AP003343, which contains six predicted genes; (d) the genomic structure of LOC_Os01g52680 (OsMADS32); deletion of an A nucleotide (C556), which disrupts a Dde I restriction site, was observed within exon 3 in the tril mutant; (e) detection of the mutated site by using a CAPS marker CHR0135.

Table 2 Comparison of some characteristics between the tril mutant and the wild type Taipei $309^{\text {a) }}$

\begin{tabular}{lccccccc}
\hline Year & Line & Plant height $(\mathrm{cm})$ & Effective tillers & Grain length $(\mathrm{mm})$ & Grain width $(\mathrm{mm})$ & Grain thickness $(\mathrm{mm})$ & 1000 -grain weight $(\mathrm{g})$ \\
\hline \multirow{2}{*}{2011} & Mutant & $108.82 \pm 2.77^{* *}$ & $8.80 \pm 1.77^{*}$ & $7.27 \pm 0.25$ & $3.95 \pm 0.20^{* *}$ & $2.71 \pm 0.07^{*}$ & $26.11 \pm 0.34^{*}$ \\
& Wild type & $122.48 \pm 4.34$ & $6.40 \pm 1.95$ & $7.75 \pm 0.14$ & $3.62 \pm 0.07$ & $2.36 \pm 0.12$ & $27.39 \pm 0.06$ \\
\hline \multirow{2}{*}{2010} & Mutant & $109.86 \pm 3.08^{* *}$ & $7.80 \pm 1.30$ & $7.16 \pm 0.15$ & $3.59 \pm 0.15$ & $2.84 \pm 0.10^{* *}$ & $23.89 \pm 0.25^{* *}$ \\
& Wild type & $115.70 \pm 1.39$ & $8.70 \pm 1.59$ & $7.48 \pm 0.31$ & $3.53 \pm 0.10$ & $2.30 \pm 0.10$ & $26.97 \pm 0.08$ \\
\hline
\end{tabular}

a) * Significance at $P<0.05$ by $t$-test; ** Significant at $P<0.01$ by $t$-test.

gene function.

The OsMADS32 gene is predicted to consist of six introns and seven exons with a 591-bp coding region (Figure 2(d)). The mutated locus in the wild type Taipei 309 encompasses the recognition site for the restriction endonuclease Dde I (5'-CTG $\left.\underline{A} G-3^{\prime}\right)$. Thus, the mutated region in the tril mutant could not be digested by Dde I due to the deletion of nucleotide $\mathrm{A}$ in the recognition sequence. Therefore, we designed a CAPS marker CHR0135 (Table 1), and a 415-bp DNA fragment was amplified from the genomic DNA of both the wild type Taipei 309 or the tril mutant. After digestion with Dde I, two small fragments of 
sizes 149-bp and 266-bp, respectively, were obtained in the wild type, but the 415-bp fragment was not digested in the tril mutant (Figure 2(e)). This further confirmed that the predicted mutation was present in the OsMADS32 gene in the tril mutant. Taken togather, the gene sequencing and the CAPS marker test strongly indicate that the OsMADS32 gene is the candidate gene for TRII.

\subsection{Expression pattern of the OSMADS32 gene in rice}

Total RNAs were extracted from different organs, cDNA was synthesized, and semi-quantitative RT-PCR was performed. The results showed that the OsMADS32 gene is highly expressed in the inflorescence, but is barely detectable in other organs including the root, culm, leaf, leaf sheath and seeds. Real-time PCR analysis further confirmed the relatively high level of expression of the OsMADS32 gene in the inflorescence, and the very low level of expression in other tissues (Figure 3(b)).

\section{Disscussion}

To date, several genes controlling rice panicle development have been reported, and most of these were identified as mutants; examples are leafty head (lhd) [14,15], branched floretless 1 (bfl1) [16], short panicle 1 (spl) [17], aberrant spikelet and panicle 1 (asp1) [18], floral organ number (fon) [6,18,19], and enclosed panicle 2 (esp2) [20]. Also, other genes have been reported to be associated with rice hull development, including frizzy panicle ( $f z p$ ) [21], abnormal hull (ah) [22], long sterile lemma ( $\mathrm{gl}$ ) [23], elongated empty glume (ele) [24], leafy hull sterile 1 (lhs1) [25], depressed palea (dp) [26], and triangular hull (thl) [11]. Some of these genes have been cloned and their functions were analyzed; for example, the thl mutant controlled by a recessive gene has an apophysis, tortuous and narrower lemma and palea [11].

In the present study, we identified a tril mutant displaying triangular hull with an apophysis lemma and palea. The phenotype of the tril mutant is similar, but not identical, to that of the thl mutant [11]. The hull of the thl mutant is tortuous and slender, while that of the tril mutant is wide, short and thick, and the middle sections of some glumes do not close completely at the stage of inflorescence development. The top of the tril lemma is severely curved inwards toward the palea, and the transverse side of the tril palea is extended somewhat. Therefore, the tril mutant displays the triangular hull phenotype, which wraps around most of the embryo and results in an abnormal grain and brown rice kernels. The results of the present study showed that, compared with the wild type, the tril mutant presents increased grain thickness, reduced plant height and 1000-grain weight, suggesting that there is a definite effect of the mutation in the target gene on plant development and grain shape.

The present study also showed that there was no obvious difference in grain length, grain width and effective tiller number between the mutant and the wild type, which suggested that the tril mutation had little effect on these three traits. The results from grain quality analyses also showed that the RVA profiles and other propterties of the tril grains were similar to those of the wild type, suggesting that the target mutation had little effect on major determinants of grain quality. While the grain protein content of the tril mutant is significantly higher than in the wild type, it could perhaps be that the abnormal hull results in an abnormal grain and affects grain filling.

In this study, the TRII gene was mapped to the long arm of chromosome 1, which showed that it was not an allele of the $t h 1$ gene. Previously, no gene related to rice hull development had been reported in this region of the rice genome; we, therefore, concluded that TRII was a new gene regulating lemma and palea development. Sequence verification indicated that the tril mutant harbored a nucleotide A deletion within the third exon of OSMADS32, leading to a predicted translational frameshift at the 107th codon, which in turn results in premature termination at the 109th codon. The results from semi-quantitative RT-PCR and real-time PCR analyses showed that the expression of OsMADS32 exhibited organ-specificity, and that the OsMADS32 gene was mainly expressed temporally during the period of inflorescence development, while the expression in root, culm leaf, leaf sheath and seed was almost undetectable. Therefore, we suggest that the OsMADS32 gene could participate (a)

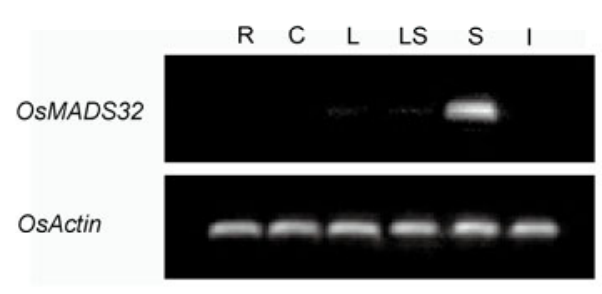

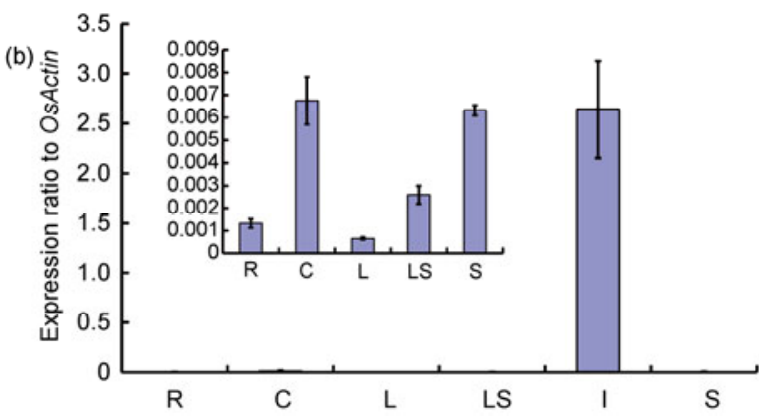

Figure 3 Expression pattern of the OsMADS32 gene in rice. (a) and (b) show the semi-quantitative RT-PCR and real-time RT-PCR results, respectively. R, root; C, Culm; L, leaf; LS, leaf sheath; I, inflorescence; S, seed. 
in the development of the rice panicle, and it is the candidate gene for TRII.

Previous research has shown that many of the genes involved in floral organ development in plants belong to the MADS-box family, such as OsMADS3, OsMADS7, OsMADS8, OsMADS13, OsMADS58, etc. The MADS-box genes comprise a large family, and exist widely in animals, plants and fungi. Each member has a highly conserved MADS-box domain sequence. Arora et al. [28] found that there were more than 75 MADS-box genes in rice, and most of them were categorized into the MIKC groups based on a genome-wide molecular characterization and microarray-based expression profiling analyses. Some studies have indicated that the MADS-box genes play an important role in rice growth and floral organ development. Kim et al. [29] reported that OSMADS51 acts to promote flowering, especially under short-days (SD), and functions upstream of Ehd1, OsMADS14, and $H d 3$ and downstream of OsGI, transmitting a signal from $O s G I$ to Ehdl. Ryu et al. [30] showed that, under long days (LD), OsMADS50 serves as a flowering activator by controlling various floral regulators such as OsMADS1, OsMADS14, OsMADS15, OsMADS18 and $H d 3 a$. These observations also implied that OsMADS5O and $O s M A D S 56$ function antagonistically through a combination of the OsLFL1 and Ehdl genes in regulating LD-dependent flowering, thus it could be inferred that $O s$ MADS56 was an LD-specific floral repressor.

Until now, little has been reported concerning the function of the OsMADS32 gene. Zhao et al. [31] identified a novel OsMADS32-like clade in monocotyledons, consisting of TaAGL14 and TaAGL15 in wheat and OsMADS32 in rice, and these three genes are the only identified members of this clade to date. Arora et al. [28] reported that there is no ortholog of the OsMADS32 gene in Arabidopsis, and confirmed that the OsMADS32 transcripts were only restricted to the early stages of panicle and seed development. Taken together, this suggests that the OsMADS32-subgroup genes are monocot-specific genes that play a role in regulating floral and seed development.

This work was supported by the National Key Basic Research and Development Project (2012CB944803), the National Natural Science Foundation of China (31071383), the Funds for Distinguished Young Scientists of Jiangsu Province (BK2012010) and Priority Academic Program Development from Jiangsu Government, China.

1 Fan C C, Xing Y Z, Mao H L, et al. GS3, a major QTL for grain length and weight and minor QTL for grain width and thickness in rice, encodes a putative transmembrane protein. Theor Appl Genet, 2006, 112: 1164-1171

2 Mao H L, Sun S Y, Yao J L, et al. Linking differential domain functions of the GS3 protein to natural variation of grain size in rice. Proc Natl Acad Sci USA, 2010, 107: 19579-19584

3 Tan Y F, Xing Y Z, Li J X, et al. Genetic bases of appearance quality of rice grains in Shanyou 63, an elite rice hybrid. Theor Appl Genet, 2000, 101: 823-829
4 Li Y B, Fan C C, Xing Y Z, et al. Natural variation in GS5 plays an important role in regulating grain size and yield in rice. Nat Genet, 2011, 43: 1266-1269

5 Li J, Qian Q, Long M, et al. Characterization of the rice floral organ number mutant fon3. J Integr Plant Biol, 2005, 47: 100-106

6 Chu H W, Qian Q, Liang W Q, et al. The FLORAL ORGAN NUMBER4 gene encoding a putative ortholog of Arabidopsis CLAVATA3 regulates apical meristem size in rice. Plant Physiol, 2006, 142: 1039-1052

7 Moon S, Jung K H, Lee D E, et al. The rice FON1 gene controls vegetative and reproductive development by regulating shoot apical meristem size. Mol Cells, 2006, 21: 147-152

8 Suzaki T, Toriba T, Fujimoto M, et al. Conservation and diversification of meristem maintenance mechanism in Oryza sativa: Function of the FLORAL ORGAN NUMBER2 gene. Plant Cell Physiol, 2006, 47: 1591-1602

9 Morinaga T, Fukushima E. Heritable characters in rice I. Abnormal mutant characters and their mode of inheritance. Bult Sci Facult Ter Kyushu Imp Univ, 1943, 10: 301-339

10 Dong F G, Xiong Z M, Qian Q, et al. Breeding near-isogenic lines of morphological markers in indica rice (in Chinese). Chin J Rice Sci, 1994, 8: 135-139

11 Li X J, Sun L J, Tan L B, et al. TH1, a DUF640 domain-like gene controls lemma and palea development in rice. Plant Mol Biol, 2011, 43: $1266-1269$

12 Zhou L H, Liu Q Q, Zhang C Q, et al. The crude protein contents in rice grain measured by two different methods and their relationship (in Chinese). J Yangzhou Univ (Agricult Life Sci Ed), 2009, 30: 6872

13 Dellaport S L, Wood J, Hicks J B. A plant DNA minipreparation: Version II. Plant Mol Biol Rep, 1983, 1: 19-21

14 Duan Y L, Wu W R, Liu H Q, et al. Genetic analysis and gene mapping of leafy head $(l h d)$, a mutant blocking the differentiation of rachis branches in rice (Oryza sativa L.). Chin Sci Bull, 2003, 48: 2201-2205

15 Xiong G S, Hu X M, Jiao Y Q, et al. LEAFY HEAD2, which encodes a putative RNA-binding protein, regulates shoot development of rice. Cell Res, 2006, 16: 267-276

16 Zhu Q H, Hoque M S, Dennis E S, et al. Ds tagging of BRANCHED FLORETLESS 1 (BFL1) that mediates the transition from spikelet to floret meristem in rice (Oryza sativa L). BMC Plant Biol, 2003, 3: 6

17 Li S B, Qian Q, Fu Z M, et al. Short panicle1 encodes a putative PTR family transporter and determines rice panicle size. Plant J, 2009, 58: 592-605

18 Yoshida A, Ohmori Y, Kitano H, et al. ABERRANT SPIKELET AND PANICLE1, encoding a TOPLESS-related transcriptional corepressor, is involved in the regulation of meristem fate in rice. Plant J, 2012, 70: 327-339

19 Suzaki T, Sato M, Ashikari M, et al. The gene FLORAL ORGAN NUMBER 1 regulates floral meristem size in rice and encodes a leucine-rich repeat receptor kinase orthologous to Arabidopsis CLAVATA1. Development, 2004, 131: 5649-5657

20 Guan H Z, Duan Y L, Liu H Q, et al. Genetic analysis and fine mapping of an enclosed panicle mutant esp2 in rice (Oryza sativa L.). Chin Sci Bull, 2011, 56: 1476-1480

21 Komatsu M, Chujo A, Nagato Y, et al. FRIZZY PANICLE is required to prevent the formation of axillary meristems and to establish floral meristem identity in rice spikelets. Development, 2003, 130: 38413850

22 Zhang Q F, Xu J D, Li Y, et al. Morphological, anatomical and genetic analysis for a rice mutant with abnormal hull. J Genet Genomics, 2007, 34: 519-526

23 Yoshida A, Suzaki T, Tanaka W, et al. The homeotic gene long sterile lemma $(G 1)$ specifies sterile lemma identity in the rice spikelet. Proc Natl Acad Sci USA, 2009, 106: 20103-20108

24 Hong L L, Qian Q, Zhu K M, et al. ELE restrains empty glumes from developing into lemmas. J Genet Genomics, 2010, 37: 101-115

25 Jeon J S, Jang S, Lee S, et al. Leafy hull sterile1 is a homeotic mutation in a rice MADS box gene affecting rice flower development. 
Plant Cell, 2000, 12: 871-884

26 Kishimoto N, Foolad M R, Etsuo S, et al. Alignment of molecular and classical linkage maps of rice, Oryza sativa. Plant Cell Reports, 1993, 12: 457-461

27 Jin Y, Luo Q, Tong H G, et al. An AT-hook gene is required for palea formation and floral organ number control in rice. Dev Biol, 2011, 359: 277-288

28 Arora R, Agarwal P, Ray S, et al. MADS-box gene family in rice: Genome-wide identification, organization and expression profiling during reproductive development and stress. BMC Genomics, 2007,
8: 242

$29 \operatorname{Kim} \mathrm{S} \mathrm{L}$, Lee S Y, Kim H J, et al. OsMADS51 is a short-day flowering promoter that functions upstream of Ehd1, OsMADS14, and Hd3a. Plant Physiol, 2007, 145: 1484-1494

30 Ryu C H, Lee S, Cho L H, et al. OsMADS50 and OsMADS56 function antagonistically in regulating long day (LD)-dependent flowering in rice. Plant Cell Environ, 2009, 32: 1412-1427

31 Zhao T, Ni Z, Dai Y, et al. Characterization and expression of 42 MADS-box genes in wheat (Triticum aestivum L.). Mol Genet Genomics, 2006, 276: 334-350

Open Access This article is distributed under the terms of the Creative Commons Attribution License which permits any use, distribution, and reproduction in any medium, provided the original author(s) and source are credited.

\section{Supporting Information}

Figure S1 Predicted coding region and deduced amino acid sequences of OsMADS32 in the mutant (tril) and the corresponding wild type (TRI1).

The supporting information is available online at csb.scichina.com and www.springerlink.com. The supporting materials are published as submitted, without typesetting or editing. The responsibility for scientific accuracy and content remains entirely with the authors. 